\title{
Glycoprotein 130 regulates bone turnover and bone size by distinct downstream signaling pathways
}

\author{
Natalie A. Sims, ${ }^{1}$ Brendan J. Jenkins, ${ }^{2}$ Julian M.W. Quinn, ${ }^{3}$ Akira Nakamura, ${ }^{3}$ \\ Markus Glatt, ${ }^{4}$ Matthew T. Gillespie, ${ }^{3}$ Matthias Ernst, ${ }^{2}$ and T. John Martin ${ }^{1,3}$ \\ ${ }^{1}$ Department of Medicine at St. Vincent's Hospital, The University of Melbourne, Fitzroy, Victoria, Australia \\ ${ }^{2}$ Ludwig Institute for Cancer Research, Colon Molecular and Cell Biology Laboratory, Royal Melbourne Hospital, \\ Parkville, Victoria, Australia \\ ${ }^{3}$ St. Vincent's Institute of Medical Research, Fitzroy, Victoria, Australia \\ ${ }^{4}$ Novartis Pharma, Basel, Switzerland
}

\begin{abstract}
The gp130-dependent cytokines, which signal through at least two intracellular pathways, regulate osteoclast and osteoblast formation. To define their roles in regulating bone mass, we analyzed mice in which gp130 signaling via either the signal transducer and activator of transcription (STAT) $1 / 3\left(g p 130^{\triangle S T A T / \triangle S T A T}\right)$ or SHP2/ras/MAPK $\left(g p 130^{Y 757 F / Y 757 F}\right)$ pathway was attenuated. In gp130 ${ }^{\triangle S T A T / \triangle S T A T}$ mice, trabecular bone volume $(\mathrm{BV} / \mathrm{TV})$ and turnover were normal, but bone length was reduced by premature growth plate closure, indicating an essential role for gp 130-STAT1/3 signaling in chondrocyte differentiation. In contrast, while bone size was normal in $g p 130^{Y 757 F / Y 757 F}$ mice, $\mathrm{BV} / \mathrm{TV}$ was reduced due to high bone turnover, indicated by high osteoclast surface/bone surface (OcS/BS) and osteoblast surface/bone surface (ObS/BS). Furthermore, generation of functional osteoclasts from bone marrow of $g p 130^{Y 757 F / Y 757 F}$ mice was elevated, revealing that while gp130 family cytokines stimulate osteoclastogenesis through the osteoblast lineage, gp130, via SHP2/Ras/MAPK, inhibits osteoclastogenesis in a cell lineage-autonomous manner. Genetic ablation of IL-6 in $g p 130^{Y 757 F / Y 757 F}$ mice exacerbated this osteopenia by reducing ObS/BS without affecting OcS/BS. Thus, while IL-6 is critical for high bone formation in $g p 130^{Y 757 F / Y 757 F}$ mice, it is not involved in the increased osteoclastogenesis. In conclusion, gp130 is essential for normal bone growth and trabecular bone mass, with balanced regulation depending on selective activation of STAT1/3 and SHP2/ras/MAPK, respectively. Furthermore, the latter pathway can directly inhibit osteoclastogenesis in vivo.
\end{abstract}

J. Clin. Invest. 113:379-389 (2004). doi:10.1172/JCI200419872.

Received for publication August 22, 2003, and accepted in revised form December 2, 2003

Address correspondence to: Natalie A Sims, Department of Medicine at St. Vincent's Hospital, 41 Victoria Parade, Fitzroy, Victoria 3065, Australia. Phone: 61-3-9288-2555;

Fax: 61-3-9288-2581; E-mail: nsims@medstv.unimelb.edu.au. Portions of this work were presented in abstract form at the 24th Annual Meeting of the American Society for Bone and Mineral Research in San Antonio, Texas, USA, on September 23, 2002 (J. Bone Miner. Res. 17:S154), and at the 1st Joint Meeting of the International Bone and Mineral Society and the Japanese Society for Bone and Mineral Research in Osaka, Japan, on June 6, 2003 (Bone 32:S87).

Conflict of interest: The authors have declared that no conflict of interest exists.

Nonstandard abbreviations used: leukemia inhibitory factor (LIF); cardiotrophin-1 (CT-1); oncostatin M (OsM); ciliary neurotrophic factor (CNTF); LIF receptor (LIF-R); OsM receptor (OsM-R); signal transducer and activator of transcription (STAT); proliferative zone width (Prol.Z.Wi); hypertrophic zone width (Hyp.Z.Wi); microtomography (micro-CT); parathyroid hormone (PTH); bone marrow macrophage precursor (BMMP); receptor activator of NF- $\mathrm{KB}$ ligand (RANKL); tartrate-resistant acid phosphatase (TRAP); multinucleated cell (MNC); MEM, $\alpha$ modification ( $\alpha$-MEM); growth plate width (G.P.Wi); growth plate closure (G.P.Cl); trabecular bone volume (BV/TV); trabecular number (Tb.N); trabecular thickness (Tb.Th); osteoclast surface/bone surface (OcS/BS); osteoblast surface/bone surface (ObS/BS); osteoid volume/bone volume (OV/BV); mineral appositional rate (MAR).

\section{Introduction}

The gp130 family of cytokines includes leukemia inhibitory factor (LIF), IL-11, IL-6, cardiotrophin-1 (CT-1), oncostatin $\mathrm{M}(\mathrm{OsM})$, and ciliary neurotrophic factor (CNTF). These cytokines signal by forming a receptor complex containing the common gp130 coreceptor signaling subunit (1). The specific components of this receptor complex depend on the nature of the bound ligand. IL- 11 and IL- 6 bind to specific $\alpha$-receptor subunits that lack their own signaling domains and are entirely dependent for intracellular signaling on the function of gp130 through gp130 $\beta$-subunit homodimers (1). In contrast, LIF, OsM, CT-1, and CNTF signal through gp130 heterodimers containing either LIF receptor (LIF-R) or OsM receptor (OsM-R) $\beta$ subunits. Formation of either gp130-containing complex results in activation of cytoplasmic Janus kinases, which phosphorylate tyrosine residues on gp130, LIF-R $\beta$, and OsM-R $\beta$ (2). Dimerization of gp130 results in activation of the signal transducer and activator of transcription (STAT) 1 and STAT3 $(3,4)$ and the SHP2/ ras/MAPK (5) signaling pathways. The use of distinct downstream signaling pathways from different intracellular regions of the same gp130 receptor subunit enables ligand- and tissue-specific activation of distinct 
sets of target genes and, thus, distinct physiologic effects of the gp130 cytokines in vivo (6).

The coordinated activities of osteoclasts (boneresorbing cells) and osteoblasts (bone-forming cells) determine the overall structure and strength of the mammalian skeleton. LIF, IL-11, IL-6, CT-1, and OsM have all been reported to stimulate osteoclastogenesis (7-9) and, in some cases, to stimulate osteoblast proliferation or differentiation (10-12) in cell culture systems. Transgenic overexpression of IL-11 results in increased osteoblast generation in vivo and ex vivo (13), yet overexpression of IL-6 leads to low levels of both osteoblast and osteoclast formation $(13,14)$. Furthermore, studies of knockout mice revealed elevated osteoclast numbers in the bones of LIF-R $\beta$ knockout mice (15), but low bone resorption and formation in the absence of IL-11R (16). Despite the shared use of receptor subunits, these cytokines are therefore likely to mediate their effects on different bone cell types through different intracellular signaling pathways.

Both bone formation and resorption are altered significantly in $g p 130^{-/-}$mice $(17,18)$. Although this mutation results in neonatal lethality, reduced trabecular bone mass is a feature of the neonatal skeleton of these mice. The finding of increased osteoclastogenesis both in vivo and in ex vivo cultures was surprising, given the previously reported capacity of gp130 cytokines to stimulate osteoclast formation in vitro (7-9). Furthermore, in wildtype cultures, osteoclastogenesis was inhibited by a gp130-neutralizing antibody in vitro (9), and bone loss in ovariectomized mice was prevented by treatment of the mice with a gp130 nonpeptide antagonist (19). The $g p 130^{-/-}$mice also demonstrated shortened limbs $(17,18)$; these bones are formed by controlled growth and mineralization of a cartilage model (endochondral ossification), and this defect implies an impairment in chondrocyte differentiation in the absence of gp130.

In this study, we sought to determine the specific contributions made by the two signaling pathways emanating from gp130 to normal osteoclastogenesis and to endochondral ossification by utilizing two gp130 "knock-in" mouse strains homozygous for mutations in gP130 at either the SHP2/ras/MAPK or the STAT1/3 activation sites. Specifically, gp130 1 STAT/ $\triangle S T A T$ mice express a knock-in mutant of gp130 with a C-terminally deleted gp130 that lacks all STAT1/3 binding and activation sites while retaining the capacity to activate SHP2/ras/MAPK (20). Conversely, gP130 $1757 F / Y 757 F$ mice express gp130 with a point mutation that selectively blocks the SHP2/ras/MAPK pathway, while STAT1/3 signaling remains intact (6).

\section{Methods}

Animals. Animals were generated as described previously

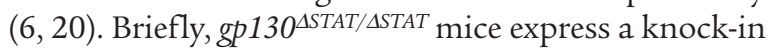
mutant of gp130 with a C-terminal deletion of the STAT1/3 binding and activation sites (20), while $g p 130^{Y 757 F / Y 757 F}$ mice express gp 130 with a point mutation that selectively blocks the SHP2/ras/MAPK pathway (6).
Compound $g p 130^{Y 757 F / Y 757 F}: I L-6^{-/-}$mice were generated from $g p 130^{Y 757 F / Y 757 F}$ mice and $I L-6^{-/-}$mice (21). All mouse strains were kept on a mixed 129/Bl6 background. For each experiment, littermate controls were used. All animal handling procedures were approved by the animal ethics committees at the Ludwig Institute for Cancer Research (Parkville, Victoria, Australia) and/or St. Vincent's Health (Fitzroy, Victoria, Australia).

Histomorphometry. Tibiae were collected from 4-weekold, 12-to 16-weeks-old, or 6-month old mice, fixed in $4 \%$ paraformaldehyde in PBS, and embedded in methylmethacrylate (22). Double-fluorochrome labeling was performed with calcein injections 10 and 3 days before tissue collection, as described previously (22). Sections 5 - $\mu \mathrm{m}$-thick sections of the proximal tibia were stained with toluidine blue or analyzed unstained for fluorochrome labels according to standard procedures using the Osteomeasure system (OsteoMetrics Inc., Decatur, Georgia, USA). Tibial cortical thickness and periosteal mineral appositional rates were measured as described previously (22). Femoral length and width were determined from contact $\mathrm{x}$-rays that were scanned and measured using NIH Image 1.62 as described previously (22). Growth plate width and thickness of the hypertrophic and proliferative zones were measured as described previously using the Osteomeasure system (OsteoMetrics Inc.) (22); proliferative zone width (Prol.Z.Wi) was defined as the width of the growth plate area in which proliferating chondrocytes were observed, and the hypertrophic zone width (Hyp.Z.Wi) was defined as the width of the growth plate in which whole lacunae containing hypertrophic chondrocytes were observed.

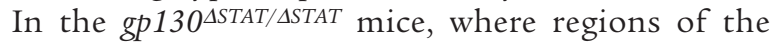
growth plate were completely closed, these parameters were measured only in those regions where cartilage was present. In addition, length of growth plate closure (complete absence of cartilage) was measured as a percentage of total growth plate length across the tibia for all genotypes using the Osteomeasure system (OsteoMetrics Inc.).

Microtomography (micro-CT). Proximal tibial bone was assessed by micro-CT measurement (microCT-20; Scanco, Bassersdorf, Switzerland) on samples from male mice $8-12$ weeks of age ( $n=7$ per group), starting $0.72 \mathrm{~mm}$ below the growth plate. A total length of $1.5 \mathrm{~mm}$ was measured at high resolution, yielding 168 sections $9 \mu \mathrm{m}$ in thickness and a voxel resolution of $9 \times 9 \times 9 \mu \mathrm{m}^{3}$. Threshold optimization was as detailed previously (23).

Serum biochemistry. Serum was collected from 10week-old mice. Serum calcium was measured by reaction with $o$-cresolphthalein (Sigma-Aldrich, St. Louis, Missouri, USA). Intact parathyroid hormone (PTH) and serum IL-6 were measured by mouse-specific ELISAs (Immunotopics, San Clemente, California, USA, and Chemicon, Temecula, California, USA).

Cell cultures. The osteoclastogenic potential of bone marrow and bone marrow macrophage precursors 

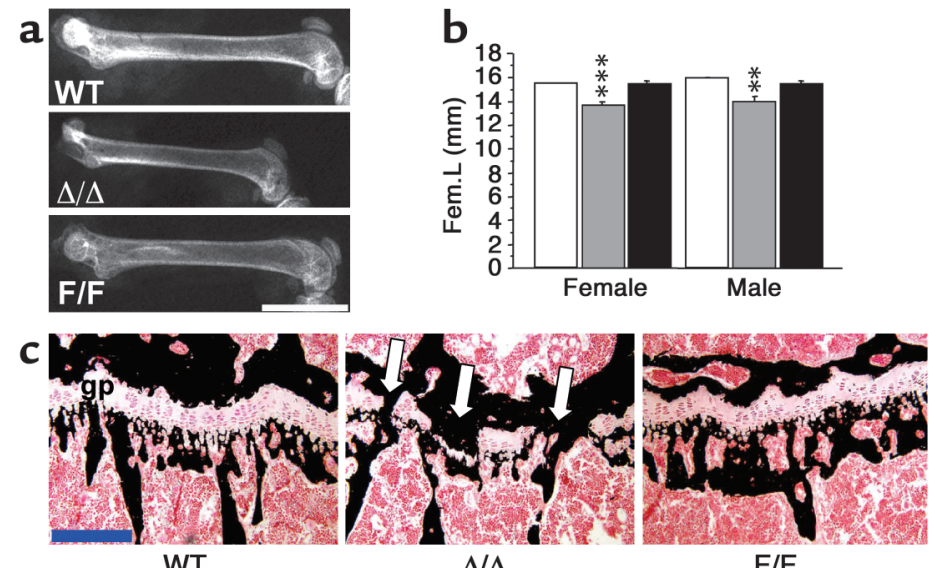

WT
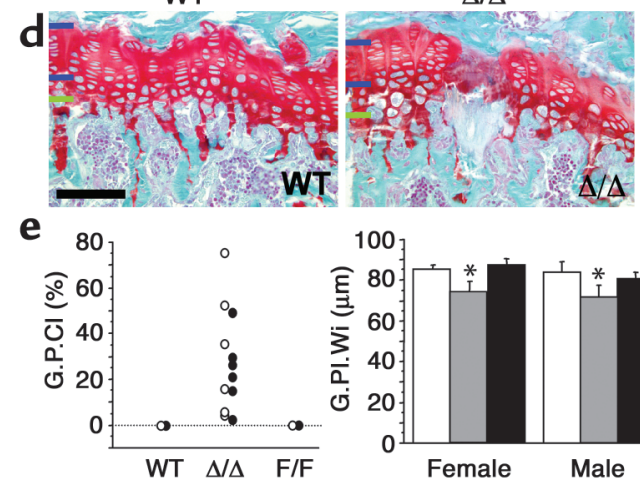

$\mathrm{F} / \mathrm{F}$
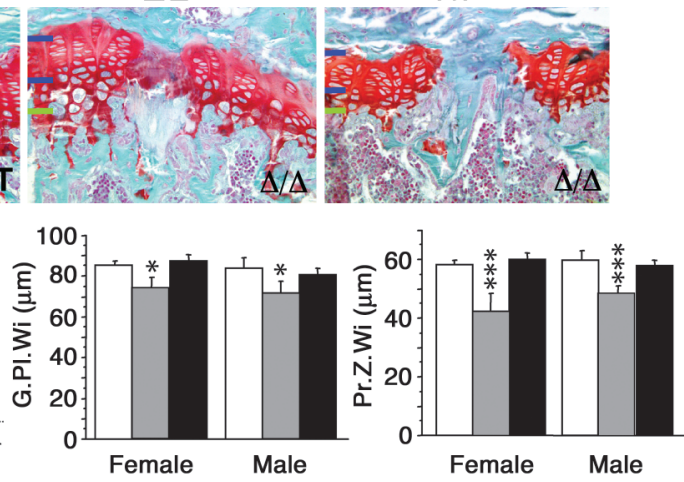

Figure 1

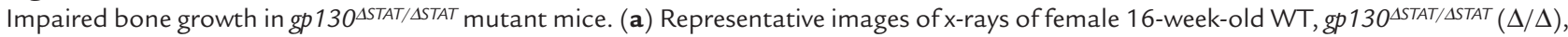
and $g$ 130 $13757 F /$ Y $757 F$ (F/F) femurs showing differences in bone size. Scale bar (white): $5 \mathrm{~mm}$. (b) Femoral length (Fem.L) in female and male WT (white bars), $\Delta / \Delta$ (gray bars), and F/F (black bars) mice. (c) Representative images of Von Kossa-stained growth plates (gp), showing calcification (black) from WT, $\Delta / \Delta$, and F/F mutant mice. Note regions of calcified bridge formation across the growth plate in $\Delta / \Delta$ (arrows). Scale bar (blue), $200 \mu \mathrm{m}$. (d) Higher-power images of Safranin O-stained growth plates (cartilage-specific mucopolysaccharides stain orange). Shown are a WT growth plate and two examples of growth plate closure in $\Delta / \Delta$ mice. Note the reduction in thickness of the proliferating zone (denoted by blue bars on the left of each micrograph), and normal hypertrophic zone thickness (lower blue bar to green bar) in the regions where the growth plate is not yet closed. Scale bar (black), $100 \mu \mathrm{m}$. (e) Percentage G.P.Cl, G.PI.Wi, and chondrocyte Pr.Z.Wi in female and male WT and gp130 mutant mice. All values are mean \pm SEM from a minimum of eight mice per group at 12-16 weeks of age. ${ }^{*} P<0.01 ;{ }^{*} P<0.01 ;{ }^{*}{ }^{*} P<0.001$ vs. WT of the same sex.

(BMMPs) was determined as described previously (24) by stimulation of bone marrow cell preparations flushed from the femora and tibiae of male and female mice (plated at a density of $10^{5}$ cells per 10mm-diameter well) with soluble recombinant GST-receptor activator of NF- $\mathrm{KB}$ ligand (RANKL) protein (25) and M-CSF (R\&D Systems, Minneapolis, Minnesota, USA). Tartrate-resistant acid phosphatase (TRAP)-positive multinucleated cells (MNCs) were counted at day 7 .

For resorption assays, bone marrow cells from gP130 $13757 F / Y 757 F$ and wild-type littermates were added to culture wells $6 \mathrm{~mm}$ in diameter $\left(10^{5}\right.$ cells/well $)$ containing $12-\mathrm{mm}^{2}$ dentine slices (26) and were stimulated with RANKL and M-CSF. After 14 days of incubation, the dentine slices were stripped of cells using $0.25 \mathrm{M} \mathrm{NH}_{4} \mathrm{OH}$, rinsed in water and ethanol, and then dried. For identification of resorption pits, the dentine slice surfaces were stained with xylene-free black ink and residual ink was removed by wiping the sur- face against absorbent paper. The proportion of the dentine surface resorbed was measured under transmission light microscopy by point counting (26).

Osteoblast differentiation was assessed with ex vivo osteoblast cultures derived from bone marrow precursors as described previously (27). Briefly, tibiae and femora from 8- to 10-week-old mice of each genotype were dissected free of adhering muscle. The ends were removed and the marrow cavity was flushed with MEM, $\alpha$ modification ( $\alpha$-MEM). Cells were plated at a density of $2 \times 10^{6}$ cells/well in 24 -well plates in $\alpha$-MEM containing 10\% FCS, $10 \mathrm{nM}$ dexamethasone, $10 \mathrm{mM} \beta$-glycerophosphate, and $50 \mathrm{mg} / \mathrm{ml}$ ascorbic acid. Cells were fixed on days 14, 21, and 28 and were stained for alkaline phosphatase to detect osteoblast colony formation or by a von Kossa technique to detect mineralization (28).

Statistical analyses. All data are presented as means \pm SEM. Significant differences were determined by oneway or two-way ANOVA followed (where significant) by Fisher's protected least significant difference post hoc 
Table 1

Serum biochemistry and cortical bone histomorphometry

\begin{tabular}{|c|c|c|c|}
\hline & Wild-type & $g p 130^{\Delta S T A T / \triangle S T A T}$ & $g p 130^{Y 757 F / Y 757 F}$ \\
\hline Serum PTH $(\mathrm{pg} / \mathrm{ml})$ & $33.1 \pm 10.6$ & $35.9 \pm 20.0$ & $54.5 \pm 13.5$ \\
\hline Serum calcium (mg/dl) & $7.35 \pm 0.12$ & $8.22 \pm 0.36$ & $7.96 \pm 0.23$ \\
\hline Serum IL-6 (pg/ml) & $5.56 \pm 1.82$ & $8.40 \pm 2.91$ & $6.41 \pm 1.52$ \\
\hline Tibial cortical thickness $(\mu \mathrm{m})$ & $113.7 \pm 3.0$ & $105.8 \pm 3.0$ & $99.3 \pm 3.1^{\mathrm{A}}$ \\
\hline Periosteal MAR ( $\mu \mathrm{m} /$ day $)$ & $0.56 \pm 0.10$ & $0.73 \pm 0.17$ & $0.50 \pm 0.13$ \\
\hline
\end{tabular}

Values are mean \pm SEM from six to eight male mice per group at 14 weeks of age. ${ }^{A} P<0.001$ vs. wildtype by one way ANOVA and Fisher's post-hoc test.

test to identify the significant pairwise differences. Where data were not normally distributed (for percentage growth plate closure measurements), significant differences were identified by the nonparametric KruskalWallis test. Simple linear regressions with ANOVA were used to determine the whether growth plate closure was associated with histomorphometric parameters reflecting growth plate chondrocyte differentiation of male and female $g p 130^{\Delta S T A T / \triangle S T A T}$ mice. For all analyses, $P<0.05$ was considered significant. All statistical analyses were carried out using StatView 5.0.1 (SAS Institute, Cary, North Carolina, USA).

\section{Results}

Mild dwarfism due to premature growth

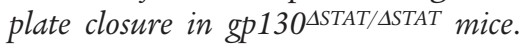
The most obvious phenotype detected in the gp130 mutants by $x$-ray analysis was a reduction in bone size in the gp130 $\triangle S T A T / \triangle S T A T$ mice (Figure 1a). Quantification revealed a significant reduction in both femoral length (12\%; Figure $1 \mathrm{~b})$ and femoral width (18\%; not shown) in both female and male gp130 $10^{\triangle S T A T / \triangle S T A T}$ mice. In contrast, gp130 ${ }^{\text {Y757F/Y757F }}$ mutant bones were normal in size (Figure 1b).

The reduced bone size in gP130 13 TAT/ $\triangle S T A T$ mice was associated with premature closure of the growth plate, the region in which chondrocyte proliferation usually determines longitudinal bone growth. In 16week-old mice, where the zone of chondrocyte prolif-
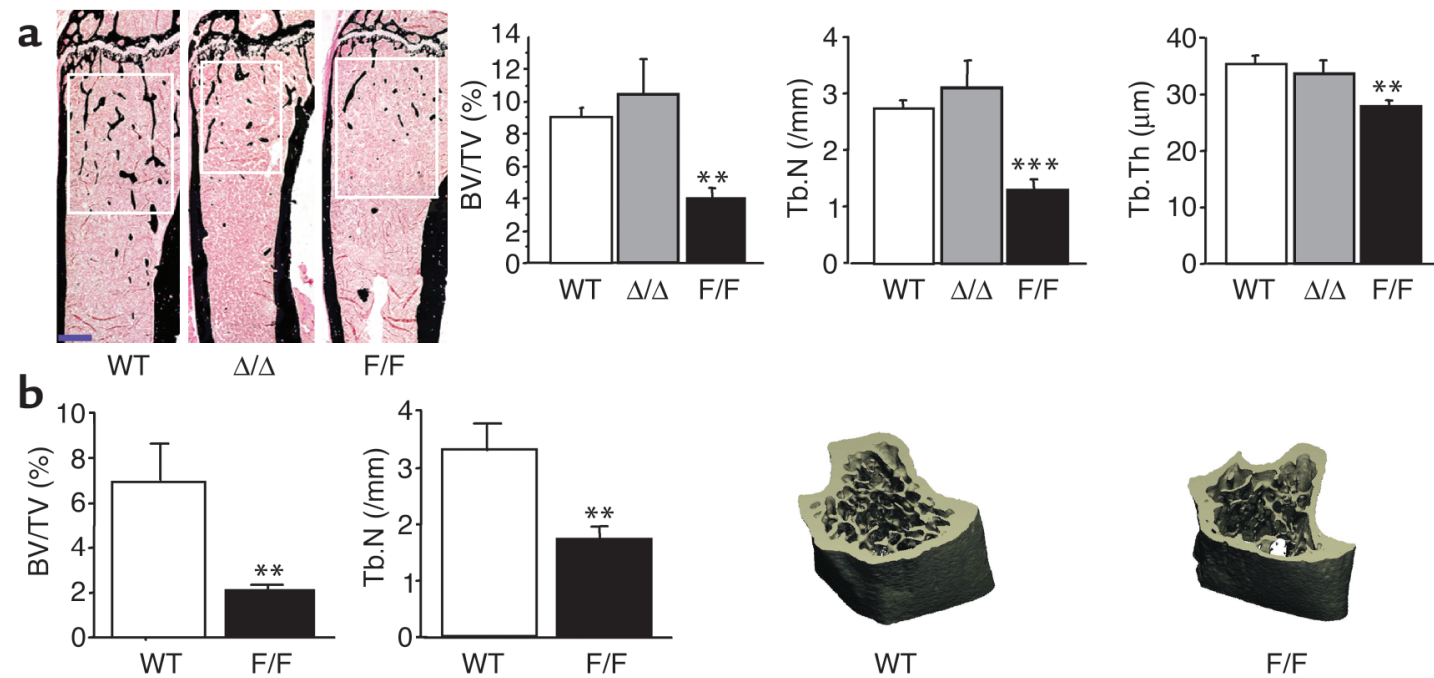

$\mathrm{F} / \mathrm{F}$
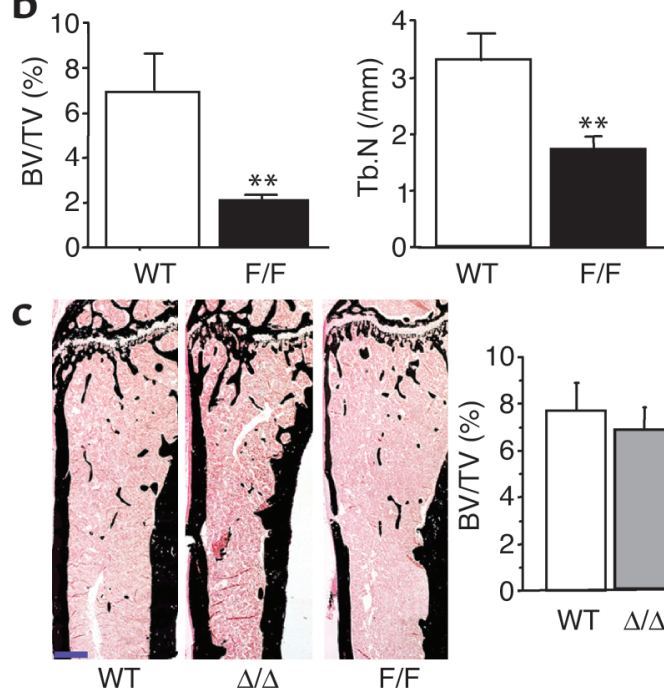

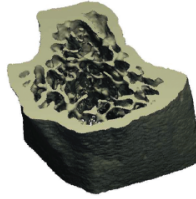

WT
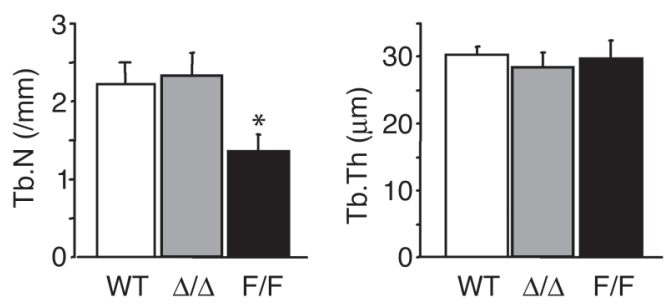

Figure 2

Low trabecular bone volume in male and female gp130 $1357 F /$ Y757F mutant mice. (a) Representative images of von Kossa-stained tibiae of male 16-week-old WT, $\Delta / \Delta$, and F/F mutant mice, showing differences in trabecular bone volume (white boxes show secondary spongiosa region used for histomorphometric measurements; note that this is a smaller anatomical region in the $\Delta / \Delta$ mice due to the smaller bone size). Scale bar (blue), $500 \mu \mathrm{m}$. Histomorphometric measures of BV/TV, Tb.N, and Tb.Th were all reduced in F/F mice compared with WT and $\Delta / \Delta$ mice. (b) Micro-CT analysis and representative three-dimensional images of tibiae from male WT and F/F mice. (c) Representative images and histomorphometry for female 16-week-old WT and gp130 mutant mice. Scale bar (blue), $500 \mu \mathrm{m}$. BV/TV is reduced in female F/F mice compared with WT and $\Delta / \Delta$ mice. All values are mean \pm SEM from a minimum of seven mice per group at $12-16$ weeks of age for histomorphometry and 8-12 weeks of age for micro-CT. ${ }^{*} P<0.05$; ${ }^{*} P<0.01$; ${ }^{*}{ }^{*} P<0.001 \mathrm{vs}$. WT of the same sex. 
eration was still clearly visible across the entire growth plate in $g p 130^{Y 757 F / Y 757 F}$ and wild-type littermates, gP130 1 STAT/ $\triangle S T A T$ mice demonstrated significant regions of growth plate closure (Figure 1, c and d). While there were no detectable regions in which the growth plate had closed in wild-type or gp130 Y757F/Y757F mice, numerous calcified bridges had formed at sites

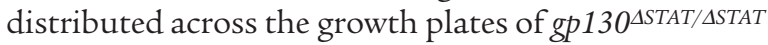
mice. Chondrocyte proliferation was not completely ceased at the time of growth plate closure in

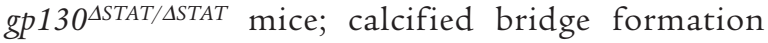
occurred adjacent to regions of continuing chondrocyte proliferation (Figure 1, c and e). Quantification revealed the total percentage of growth plate closure was similar in male and female gp130 ${ }^{\triangle S T A T / \triangle S T A T}$ mice, although this varied widely across the sample size (Figure 1e; mean values: female gp130 ${ }^{\Delta S T A T / \triangle S T A T}$ clo-

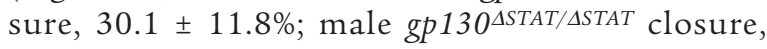
$22.6 \pm 6.6 \%$; both $P<0.001$ vs. wild-type values of 0 for all animals). In $g p 130^{\Delta S T A T / \triangle S T A T}$ mice, growth plate width (G.P.Wi) was reduced, and this was identified as a specific reduction in the Prol.Z.Wi (Figure 1e), while the Hyp.Z.Wi was not significantly altered in either sex (mean $\mu \mathrm{m} \pm$ SEM: females: wild-type, $25.0 \pm 1.4$;

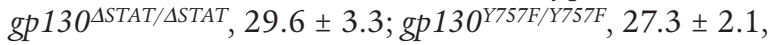
$P=0.31$; males: wild-type, $23.9 \pm 2.7 ; g p 130$ STTAT/LSTAT, $\left.23.1 \pm 4.3 ; g p 130^{Y 757 F / Y 757 F}, 22.7 \pm 1.7 . P=0.94\right)$. G.P.Wi and Prol.Z.Wi were both significantly correlated with the percentage of growth plate closure (G.P.Cl) in the

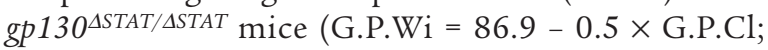
$P=0.011 ;$ Prol.Z. Wi $=53.1-0.3 \times$ G.P.Cl; $P=0.04)$, while Hyp.Z.Wi did not. There was no change in either growth plate width or widths of either the hypertrophic or proliferating zones in $g p 130^{Y 757 F / Y 757 F}$ mice (Figure 1 and results presented above). Neither cortical thickness nor periosteal apposition rate were altered in 16-week-old gp130 $0^{\Delta S T A T / \triangle S T A T}$ mice (Table 1), indicating that the small bone size in these mice did not relate to impaired periosteal growth. Our data therefore indicate that the presence of the gp130dependent STAT $1 / 3$ signaling pathway, but not the SHP2/ras/MAPK pathway, plays an essential role in determining normal bone size by regulating chondrocyte proliferation.

High bone turnover and osteopenia in gp130Y757F/Y757F mice. Despite the reduced bone size, trabecular bone volume (BV/TV) in adult male and female g $130^{\triangle S T A T / \triangle S T A T}$ mice was not significantly altered (Figure 2 , a-c). In contrast, both male and female $g p 130^{Y 757 F / Y 757 F}$ mice demonstrated a significant reduction in $\mathrm{BV} / \mathrm{TV}$, as initially suggested by $x$-ray (Figure 1a) and subsequently confirmed by both two-dimensional histomorphometry and three-dimensional micro-CT analysis (Figure 2, a-c). In adult male gp130 $1757 F / Y 757 F$ mice, $\mathrm{BV} / \mathrm{TV}$ and trabecular number (Tb.N) were halved, and trabecular thickness (Tb.Th) was significantly reduced, compared with wildtype littermates (Figure 2, a and b). In female mice of the same age, the effect was less dramatic and was not detected in $\mathrm{Tb}$.Th, probably because, as is usual for female mice (29), BV/TV was already lower in females than in males (Figure 2c). Low BV/TV was also detected in 4-week-old and 6-month-old gp130 $13757 F / Y 757 F$ mice a
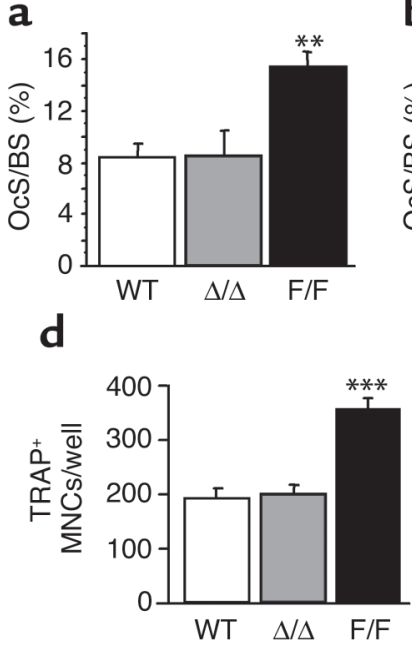

b

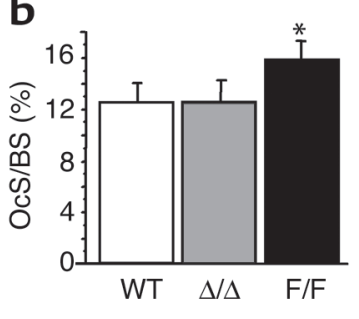

e

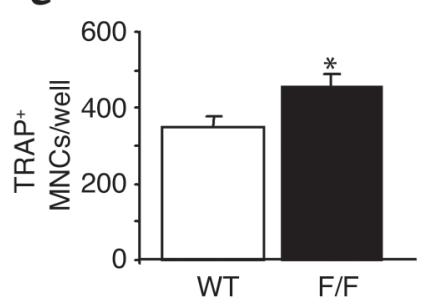

c

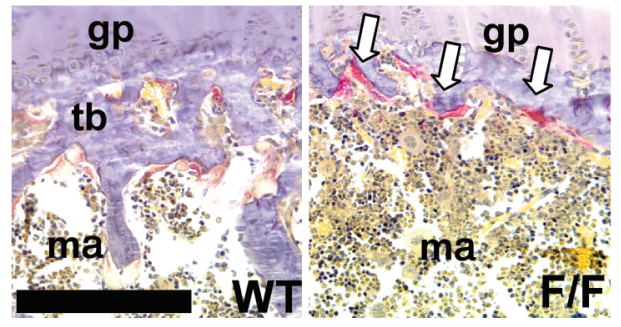

f

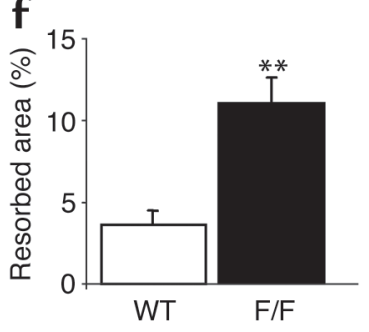

\section{Figure 3}

Increased osteoclast formation in male and female gp130 Y757F/Y757F mutant mice. Tibial trabecular OcS/BS was significantly increased in male (a) and female (b) F/F mice compared with WT and $\Delta / \Delta$ mice. All values are mean \pm SEM from a minimum of eight mice per group at $12-16$ weeks of age. ${ }^{*} P<0.05 ;{ }^{*} P<0.01 ;{ }^{*}{ }^{*} P<0.001 \mathrm{vs}$. WT of the same sex. (c) Representative images of TRAP-stained primary spongiosa from WT and F/F mice. Note the large number of TRAP-positive osteoclasts (arrows) and the absence of trabecular bone in the F/F section. gP, growth plate; m, marrow; tb, trabecular bone. Scale bar (black), $200 \mu \mathrm{m}$. (d and e) In vitro osteoclastogenesis assays demonstrating increased TRAP-positive MNCs (TRAP ${ }^{+}$MNCs/well) generated from RANKL-induced primary bone marrow cultures (d) and BMMP cultures (e) from WT and gp130 mutant mice. (f) Pit formation by osteoclasts generated from RANKL-induced primary bone marrow cultures from WT and gp130 mutant mice. All values are mean \pm SEM of quadruplicate cultures from four separate mouse bone marrows of mixed sexes. ${ }^{*} P<0.05 ;{ }^{*} P<0.01 ;{ }^{*}{ }^{*} P<0.005$ vs. WT culture. 

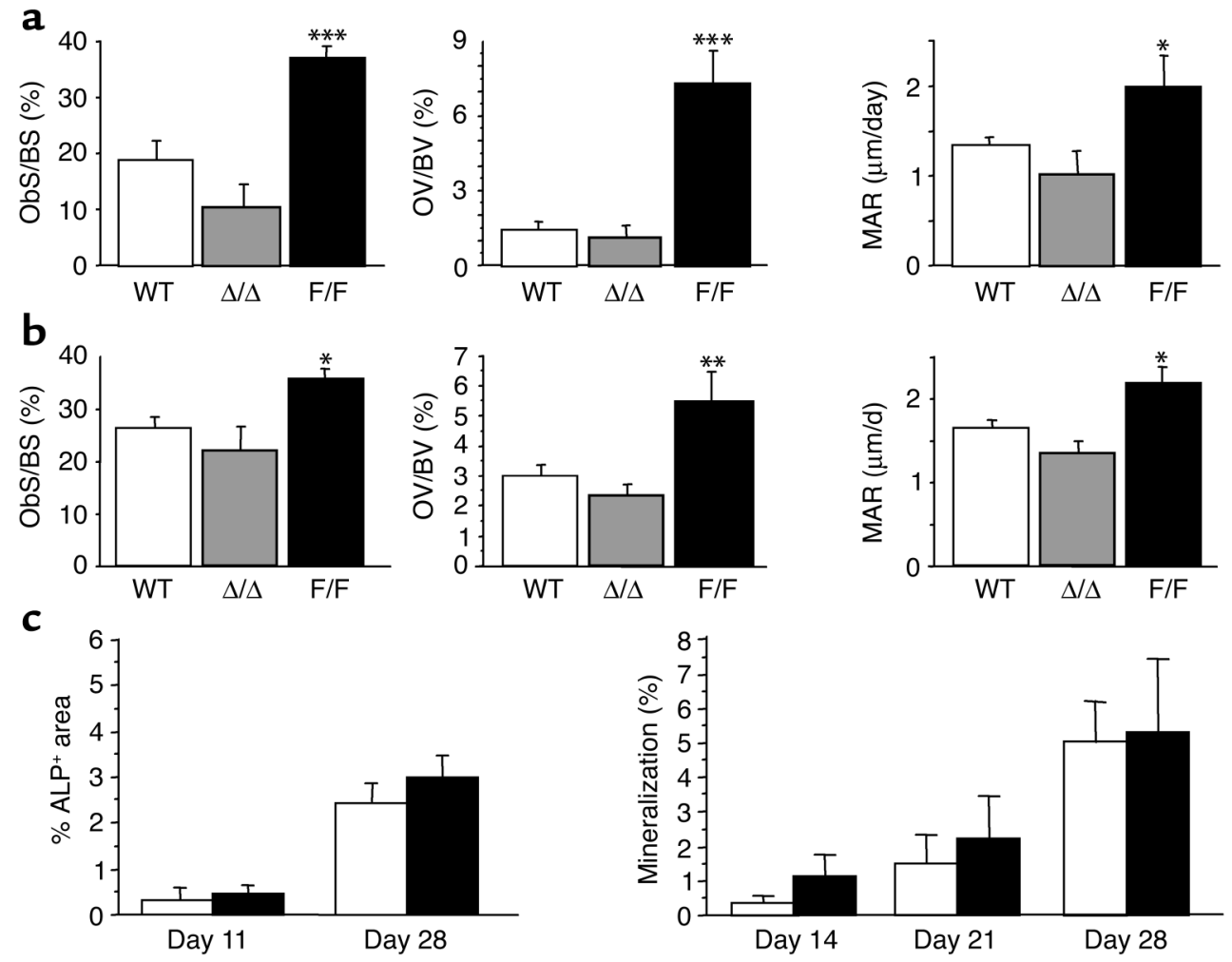

Figure 4

Increased bone formation in male and female gp 130 Y757F/Y757F mutant mice. Histomorphometric indices of bone formation including tibial $\mathrm{ObS} / \mathrm{BS}, \mathrm{OV} / \mathrm{BV}$, and MAR were significantly higher in male (a) and female (b) F/F mice compared with WT and $\Delta / \Delta$ mice. All values are mean \pm SEM from a minimum of eight mice per group at $12-16$ weeks of age. ${ }^{*} P<0.05 ;{ }^{*} P<0.01 ;{ }^{*}{ }^{*} P<0.001 \mathrm{vs}$. WT of the same sex. (c) Ex vivo osteoblast differentiation from bone marrow cultured under osteoblastogenic conditions was not significantly altered in $\mathrm{F} / \mathrm{F}$ mice (black bars) compared with WT mice (white bars). Shown are alkaline phosphatase-positive colony formation as a percentage of well area $\left(\% \mathrm{ALP}^{+}\right.$area $)$and mineralization detected by a von Kossa stain for calcified matrix (\%). Values are mean \pm SEM from three experimental preparations at each time point using a minimum of three mice of mixed sexes per genotype for each experiment.

compared with wild-type littermates (data not shown). These observations indicate that the gp130-dependent SHP2/ras/MAPK pathway is essential for the maintenance of normal trabecular bone structure.

The low BV/TV observed in the gp130 $1757 F / Y 757 F$ mice was associated with high osteoclast numbers in vivo. Within the secondary spongiosa, where bone is remodeled by repeated cycles of bone resorption and formation, the percentage of trabecular bone covered by osteoclasts (osteoclast surface/bone surface; OcS/BS) (Figure 3a) and the number of osteoclasts (not shown) were approximately doubled in the secondary spongiosa of male mice and, even in females, in which bone turnover is usually higher than in males (29), OcS/BS and osteoclast numbers were also increased when the SHP2/Ras/MAPK pathway was mutated (Figure 3b). In the $g P 130^{Y 757 F / Y 757 F}$ mice, a large number of active osteoclasts was also detected at the base of the growth plate, where new trabecular bone is normally generated (Figure 3c). No change in osteoclast surface or number was detected in either male or female $g p 130^{\triangle S T A T / \triangle S T A T}$ mice (Figure 3 , a and b). Cortical thickness was also reduced in $g p 130^{Y 757 F / Y 757 F}$ mice (Table 1). Since the level of periosteal mineral apposition (trans- verse bone growth) was not altered in these mice (Table 1), this supports the finding of elevated osteoclast activity in $g p 130^{Y 757 F / Y 757 F}$ mice.

Because elevated bone resorption in vivo can be associated with alterations in calcium homeostasis, we measured serum calcium and circulating PTH levels. Despite the high level of bone turnover, there was no significant alteration in either of these parameters in the gp130 mutants (Table 1), as has been observed in other animal models of high bone turnover osteopenia $(30,31)$, suggesting that the increased osteoclastogenesis is not secondary to altered PTH signaling or impaired calcium homeostasis.

Increased osteoclastogenesis in $g p 130^{\text {Y757F/Y757F mice }}$ was reproduced in ex vivo cultures of bone marrow cells derived from these mice. While RANKL induced a similar level of osteoclast formation by wild-type and gp130 1 STAT/ASTAT bone marrow, there was a significant elevation in osteoclastogenesis from RANKLstimulated $g p 130^{Y 757 F / Y 757 F}$ bone marrow (Figure 3d). When a more homogeneous preparation of osteoclast precursors from the marrow (BMMPs) was used, there was again a significant elevation in osteoclastogenesis from $g p 130^{Y 757 F / Y 757 F}$ compared with wild- 
a
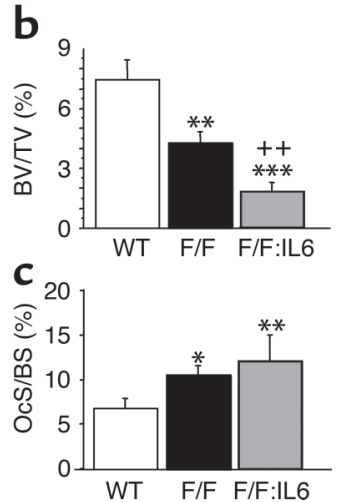
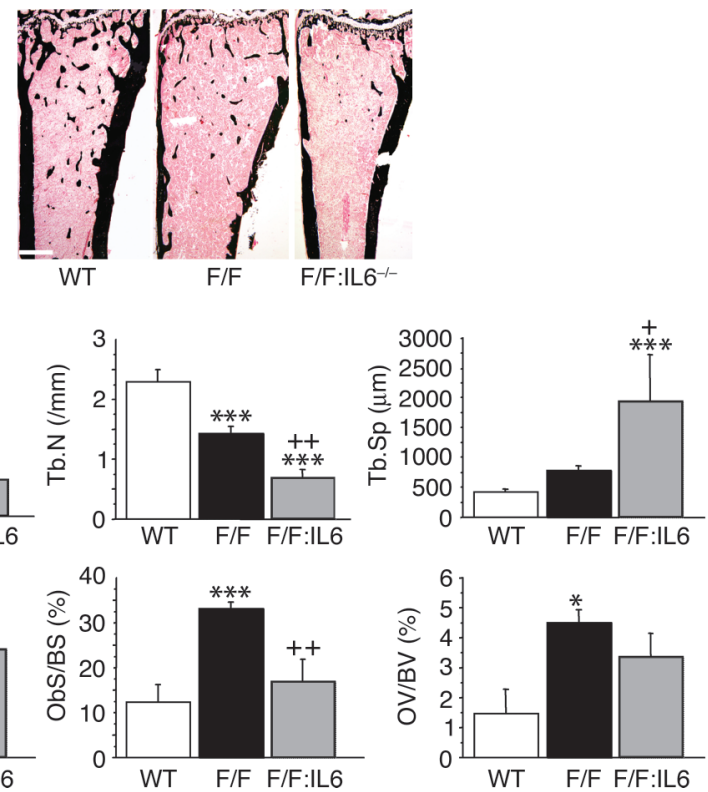

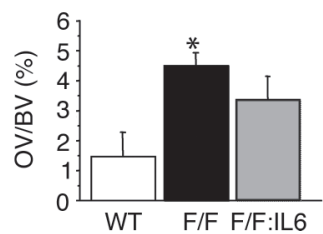

\section{Figure 5}

IL-6 contributes to the high level of bone formation in gp130 Y757F/Y757F mutant mice. (a) Representative images of von Kossa-stained tibiae from 16 week-old WT, F/F, and F/F:IL-6-/- (F/F:IL-6) mice. Scale bar (white), $500 \mu \mathrm{m}$. (b) BV/TV and Tb.N are reduced further in F/F:IL-6-/- mice compared with WT and F/F mice. Tb.Sp, trabecular separation. (c) Deletion of IL- 6 rescues the high ObS/BS and reduces the OV/BV of F/F mutant mice but not the high OcS/BS. All values are mean \pm SEM from six female mice per group at 14-16 weeks of age. ${ }^{*} P<0.05 ;{ }^{*} P<0.01 ;{ }^{*} P<0.001$ vs. WT; ${ }^{++} P<0.01,{ }^{+} P<0.05$ vs. F/F. type precursors (Figure 3e). The ability of these osteoclasts to resorb bone was confirmed by pit assays, demonstrating an increase in area resorbed by osteoclasts derived from $g p 130^{Y 757 F / Y 757 F}$ marrow of a similar proportion to the increase in osteoclast numbers (Figure 3f). These data confirm that the defect in osteoclast number observed in vivo is not mediated by systemic hormones or altered paracrine signaling from osteoblasts but instead is autonomous within the hemopoietic cell lineage.

As observed in many osteopenic states, the high level of bone resorption in $g p 130^{Y 757 F / Y 757 F}$ mice was associated with a high level of bone formation. Histomorphometric markers of bone formation were significantly elevated in both male and female $g p 130^{Y 757 F / Y 757 F}$ mice. These included osteoblast surface/bone surface (ObS/BS), indicating a greater number of osteoblasts on trabecular bone surfaces; osteoid volume/bone volume $(\mathrm{OV} / \mathrm{BV})$, indicating a high level of new bone matrix (osteoid) production; and mineral appositional rate (MAR), indicating a high rate of mineralization of the bone matrix (Figure 4, a and b). We also observed increases in osteoid surface, osteoid thickness, and the number of osteoblasts on trabecular surfaces in both male and female $g p 130^{Y 757 F / Y 757 F}$ mice (data not shown). However, when osteoblast precursors were grown ex vivo from wild-type and gp130 $1757 F / Y 757 F$ bone marrow cells, we did not observe any significant difference in the ability of these precursors to differentiate into alkaline phosphatase-positive cells (osteoblasts) or any change in their ability to form mineralized nodules (Figure 4c). This indicated that the high level of bone formation in $g p 130^{Y 757 F / Y 757 F}$ mice was not cell autonomous but may result from altered paracrine or endocrine activation taking place in the mutant mice. No change in any marker of bone formation was observed in $g p 130^{\triangle S T A T / \triangle S T A T}$ mutants (Figure 4).
Osteoclastogenesis phenotype is independent of IL-6, but that of the osteoblast is IL-6 dependent. Because IL-6 is known to stimulate osteoblast differentiation and osteoclastogenesis (32-35) and IL-6-stimulated STAT3 activation is enhanced in $g p 130^{Y 757 F / Y 757 F}$ mice (6), we next set out to determine whether the increased bone formation and bone resorption in gp130 $0^{Y 757 F / Y 757 F}$ mice were mediated by IL-6. To do this, we carried out bone histomorphometry on adult gp130 Y757F/Y757F: $1 \mathrm{~L}-6^{-/-}$mice. Surprisingly, the genetic ablation of IL-6, which itself does not lead to significant bone abnormality in the basal state (36), led to a further reduction in bone mass, indicated by BV/TV and Tb.N, as well as an increase in trabecular separation in compound mutant (gp130 Y757F/Y757F:IL-6-/-) mice compared with single mutant gp130 $13757 F / Y 757 F$ mice, irrespective of sex (Figure 5 shows results from female mice only). While OcS/BS remained high in the compound mutants, the high level of ObS/BS observed in $g p 130^{Y 757 F / Y 757 F}$ mice was blocked and the high OV/BV was no longer significantly elevated in the absence of IL-6 (Figure 5), suggesting that IL- 6 mediates the moderating effect of the gp130-dependent SHP2/ras/MAPK pathway on bone formation but not on osteoclastogenesis.

\section{Discussion}

The gp130-family of cytokines, including IL-6, IL-11, LIF, OsM, and CT-1, have been implicated in the determination of bone size and bone density from their effects in bone cell culture systems $(9,32,33)$ and from the skeletal defects of transgenic $(13,14)$ and knockout mice $(15,16,36)$. The contributions of the major intracellular gp130 signaling pathways responsible for bringing about these biological observations remain unknown. Here we have used knock-in gp130 mutant mice unable to elicit either gp130-dependent STAT1/3 or SHP2/ras/MAPK activation in order to detail the 
specific involvement of these pathways in regulating physiological bone turnover in vivo. This analysis allows us to define three distinct pathways that regulate bone cell function in vivo in response to gp130 family cytokines: (a) gp130-STAT1/3 regulation of chondrocyte proliferation, (b) gp130-SHP2/Ras/MAPK inhibition of osteoclastogenesis, and (c) IL-6 stimulation of osteoblasts through the gp130-STAT1/3 pathway (these pathways are summarized in Figure 6).

It has been suggested that gp130 family cytokines, particularly IL-6 and IL-11, may be involved in regulating sex steroid-mediated effects on bone growth and trabecular bone structure $(12,37)$. In this study, we observed an identical response in male and female mice to both gp130 mutations, suggesting that gp130 family members are not required for sex steroid-regulated differences in bone growth and bone turnover in the basal state.

Reduced bone size, premature growth plate closure, and reduced chondrocyte proliferation in gp $130^{\triangle S T A T / \triangle S T A T}$ mice indicate that the gp130-dependent STAT1/3 signaling pathway plays an essential role in determining bone size, specifically by promoting growth plate chondrocyte proliferation. In gP130 1 STAT/ $\triangle S T A T$ mice, regions of growth plate closure were detected as early as 12 weeks of age, which is very unusual, as it is rare to detect growth plate closure in mice even at 12 months of age.

It has been reported previously that the joints of some gp130 1 STAT/ $/ S T A T$ mice exhibit excessive articular cartilage growth and joint stiffness (20); however, samples used in our study did not demonstrate this phenotype. Even if an early, undetected form of the articular cartilage defect were present in these mice, it is unlikely that this would also be a mechanism for growth plate closure (the opposite effect) in an area removed from the synovial environment. Indeed, in the only reported case we have found in which changes in cytokine levels at the joint have affected both articular and growth plate cartilage (38), the changes at the growth plate were restricted to the periosteal edge (the most external part of the growth plate). In contrast, regions of growth plate closure in the gp130 ${ }^{\triangle S T A T / \triangle S T A T}$ mice occurred apparently at random across the width of the growth plate, with no growth plate closure being observed at the periosteal edge.

The mechanistic details of growth plate closure are not well understood in mammals, with part of the difficulty being that full growth plate closure, which is observed in adult humans, is not reported in rodents; thus, there is no good animal model available for the study of growth plate closure. Generally, it has been understood that growth plate closure occurs along with a gradual reduction in chondrocyte proliferation and is characterized by osteoclastic resorption of quiescent cartilage (39). Although reduced chondrocyte proliferation was observed in $g p 130^{\triangle S T A T / \triangle S T A T}$ mice and was correlated significantly with the extent of growth plate closure, we were surprised to find active chondrocyte proliferation immediately adjacent to regions
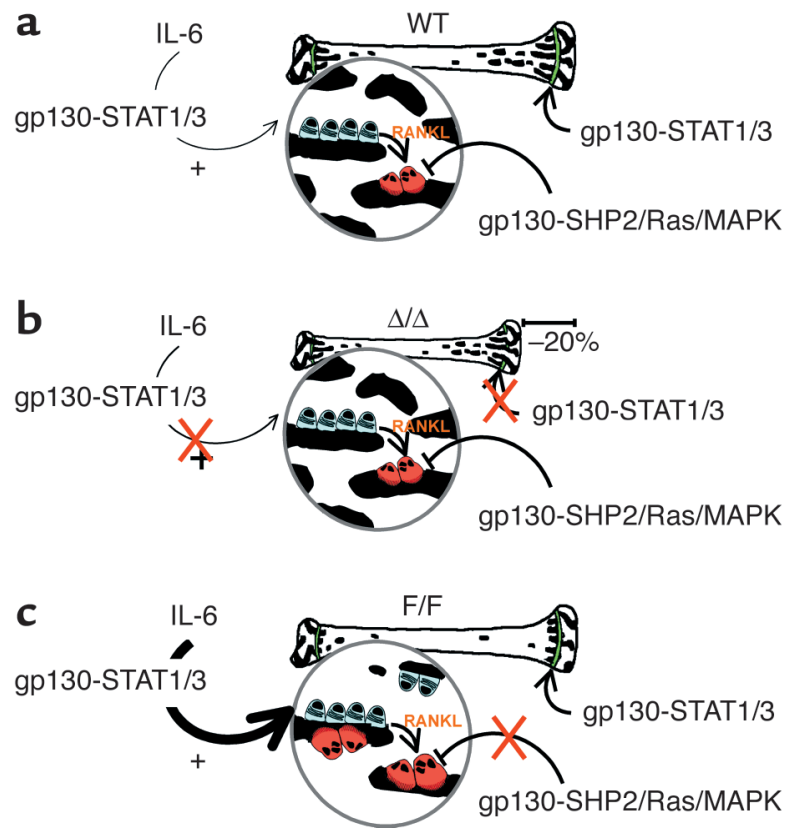

\section{Figure 6}

Summary of the effects of gp130 signaling pathway mutations on bone structure and bone cell function. (a) In WT mice, IL-6 stimulates osteoblast proliferation via the gP $130-$ STAT1/3 pathway, and the osteoblasts in turn stimulate osteoclastogenesis by the RANKL pathway. Osteoclastogenesis is also inhibited by gP130 family cytokines, acting through the gP130-SHP2/Ras/MAPK signaling pathway within the hematopoietic lineage. Chondrocyte proliferation is stimulated by gp 130 family cytokines through the gp 130 STAT1/3 pathway. (b) In the absence of the gp130-induced STAT1 $/ 3$ signaling pathway, indicated by the red $X$ (in $\Delta / \Delta$ mice), chondrocyte proliferation is no longer stimulated through the gP130-STAT1/3 pathway, leading to reduced bone length. Inhibition of osteoclastogenesis through the gP130-SHP2/Ras/MAPK pathway remains intact, and while IL-6 stimulation of osteoblast differentiation can no longer occur through the gP130-STAT1/3 pathway, this is clearly a minor pathway not required for the normal level of bone formation, as bone turnover and bone structure remain at the same level as in WT mice. (c) In F/F mice, IL-6-stimulated osteoblast proliferation via gP130-STAT1/3 is enhanced, leading to a greater number of osteoblasts. Deletion of the gP130SHP2/Ras/MAPK pathway, indicated by the red X, releases inhibition, resulting in increased osteoclastogenesis. The increase in resorption appears to be greater than the increase in bone formation, leading overall to a reduction in trabecular bone density.

of full growth plate closure (calcified bridge formation) in the gp130 13 STAT/ $\triangle S T A T$ mice. This is consistent with a recent report of calcified bridge formation across the growth plate in rats as young as 3 months of age, although that study did not report the level of chondrocyte proliferation (40). Clearly, there is some mechanism whereby growth plate closure can occur without full cessation of chondrocyte proliferation, although we cannot say whether the effect observed in our study is physiological or pathological. Nevertheless, this suggests a heterogeneous response of the chondrocyte population to the absence of STAT $1 / 3$ signaling downstream of gp130. 
While it has been reported previously that gp130 family members, particularly IL- 6 and OsM, regulate articular cartilage breakdown and may play a role in the pathogenesis of various aspects of arthritis (41, 42), gp130 family members have not been studied in growth plate chondrocytes or in longitudinal bone growth. The LIF-R $\beta$ knockout mouse exhibits reduced postnatal skeletal growth (15), but changes in the growth plates of these mice have not been reported. As bone size is normal in IL-6 and IL-11R $\alpha 1$ knockout mice (16), the reduced bone size observed in $g p 130^{\Delta S T A T / \triangle S T A T}$ mice may relate to impaired STAT1/3-mediated signaling in response to gp130:LIF-R $\beta$ heterodimerization. LIF, acting via LIF-R $\beta$ and the STAT $1 / 3$ pathway, may play an essential role in maintaining a high level of chondrocyte proliferation during skeletal growth.

No change in osteoblast or osteoclast activity or trabecular bone volume was detected when gp130dependent STAT1/3 signaling was abolished in gp130 1 STAT/ $\triangle S T A T$ mutants, suggesting that STAT $1 / 3$ is not required for normal bone turnover in the basal state, despite its involvement in the generation of RANKL in response to gp130 cytokines by osteoblastic stromal cells in vitro (43). In contrast, the gP $130^{Y 757 F / Y 757 F}$ mutant mice demonstrated high levels of both bone formation and bone resorption, resulting in an osteopenic phenotype. The increased osteoclast number, but not the increased osteoblast number, observed in the gP $130^{Y 757 F / Y 757 F}$ mutant mice was reproduced in ex vivo cultures of bone marrow cells from these mice. Here we saw that formation of functional osteoclasts induced by the same dose of RANKL was dramatically elevated in the $g p 130^{Y 757 F / Y 757 F}$ mice but was not changed in $g p 130^{\triangle S T A T / \triangle S T A T}$ mice. This result suggests that the defect in osteoclast number observed in vivo may relate to an inherent change in the osteoclast precursor population and is not mediated by systemic hormones or altered paracrine signaling from osteoblasts. Since these mice also display increased numbers of macrophage CFU progenitors (44), which themselves are able to give rise to osteoclasts, it appears likely that the altered number of osteoclast progenitors in the bone marrow population used for the ex vivo cultures may cause the high level of bone resorption in vivo. To account for this inherent variation in the precursor population, we examined the osteoclastogenic potential of a more homogeneous population of osteoclast precursors (BMMPs). Even in this preparation, in which osteoclast precursor numbers are equal for both genotypes, the precursors from $g p 130^{Y 757 F / Y 757 F}$ mutant continued to show a heightened osteoclastogenic response to RANKL. Thus, deletion of the gp130SHP2/Ras/MAPK pathway gives rise to a greater number of osteoclast precursors, and these precursors have a greater maximum response to RANKL, leading to increased osteoclastogenesis.

As a high level of osteoclastogenesis is also observed in $L I F-R \beta^{-/}$and $g p 130^{-/-}$mice $(15,17,18)$, our study has demonstrated a novel role for gp130 in the osteoclast cell lineage in controlling osteoclastogenesis. It supports a model whereby gp 130 family members signal through the gp130-SHP2/ras/MAPK pathway to limit osteoclast formation and indicates that this property is contained within the hematopoietic lineage. Previously, it had been recognized that activation of gp130 on osteoblasts by IL- 6 indirectly stimulated osteoclast formation in vitro $(9,32,43)$ via activation of STAT3 (34). Although STAT3 activation is upregulated in gp130 Y757F/Y757F mice, we know that this IL-6-dependent pathway alone is not responsible for the high osteoclastogenesis in these mice, as this is retained in gP130 $13757 F / Y 757 F: I L-6^{-/-}$mice, and osteoclastogenesis is not altered when the gp130-STAT1/3 pathway is abolished in the gP130 1 STAT/ $/$ STAT mice. Hence, our data suggests a dual role for gp130 in osteoclastogenesis based on its simultaneous expression on osteoblast and osteoclast precursors (45-47), which may be important in balancing the opposing activities of these two cell types. Although gp130 cytokines stimulate osteoclastogenesis through the osteoblast lineage, the dominant effect on bone in vivo is the hematopoietic lineage-specific effect of the gp130 pathway in inhibiting osteoclastogenesis (see Figure 6).

The Ras/MAPK pathway not only functions downstream of gp130 but also has been implicated in RANKL, IL-4, and TNF- $\alpha$ stimulation of osteoclastogenesis from bone marrow macrophage precursors (48-50). While the Ras/MAPK pathway is often implicated in the stimulation of cell proliferation and differentiation, this pathway is also involved in cell senescence, depending both on the manner of its activation and on autocrine/paracrine signaling pathways downstream of Ras/MAPK itself (51). It is likely that the downstream effectors of the MAPK pathway through which gp130 inhibits osteoclastogenesis described here are different from the stimulatory pathway previously described in osteoclastogenic cell culture systems.

Because IL- 6 stimulates both bone formation and resorption, and mutation of the SHP2 binding site enhances IL-6 signal transduction (6), we examined whether increased bone formation and resorption in gP $130^{Y 757 F / Y 757 F}$ mice is mediated by altered IL- 6 signal-

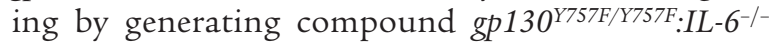
mice. Whereas $I L-6^{-/-}$mice have no bone abnormality $(16,36), \mathrm{ObS} / \mathrm{BS}$ was rescued to levels of wild-type mice in compound $g p 130^{Y 757 F / Y 757 F}:{\mathrm{IL}-6^{-/-}}^{-}$mice, while OcS/BS remained high. This high level of bone resorption without a compensatory increase in bone formation explains the very low bone mass in the compound mutant mice. It also shows that IL- 6 is not responsible for the increased osteoclast formation and bone resorption in those mice, as suggested by the ex vivo studies discussed above.

Furthermore, since gp $130^{Y 757 F / Y 757 F}$ marrow cultures did not exhibit increased osteoblast differentiation or mineralization, the high level of bone formation in $g P 130^{Y 757 F / Y 757 F}$ mice must require paracrine or endocrine signaling. The rescue of the high $\mathrm{ObS} / \mathrm{BS}$ in 
$g p 130^{Y 757 F / Y 757 F}: I L-6^{-/-}$mice is consistent with a requirement for IL-6 for the enhanced osteoblastic response in gp130 Y757F/Y757F mice. Since circulating IL-6 levels are unchanged in the gp130 $13757 F / Y 757 F$ mutants, this effect is likely to be determined by heightened intracellular STAT $1 / 3$ signaling in response to IL- 6 , as reported in the liver (6). While the IL-6-STAT1/3 pathway may be important in the high bone turnover state of the gp130 Y757F/Y757F mouse, this pathway is clearly not required for normal basal bone formation, since this is normal in $g p 130^{\Delta S T A T / \triangle S T A T}$ mice. Furthermore, this confirms cell culture studies reporting that while IL- 6 stimulation of bone formation can be mediated by either gp130 signaling pathway (34), the gp130-STAT1/3 pathway is the major pathway by which IL-6 stimulates osteoblast differentiation (35). Finally, all of this is consonant with our in vitro result that increased osteoclastogenesis in $g p 130^{Y 757 F / Y 757 F}$ mice does not result from the high level of bone formation and is independent of the effect of this mutation on osteoblasts.

In conclusion, we have defined three key pathways by which gp130 signaling contributes to bone cell interactions and to the determination of normal bone size and bone density (Figure 6a). First, gp130 cytokines, via the STAT1/3 pathway, play an essential role in stimulating chondrocyte proliferation; in the absence of this pathway, premature growth plate closure and reduced bone size is observed (Figure 6b). Second, the gp130SHP2/Ras/MAPK pathway plays an essential role in inhibiting osteoclastogenesis; in the absence of this pathway, a high level of osteoclastogenesis leads to trabecular and cortical bone loss (Figure 6c). Finally, we confirmed that IL- 6 stimulates osteoblast generation through the gp130-STAT1/3 pathway and showed that this pathway is upregulated and responsible for the high level of bone formation in the absence of the gp130-SHP2/Ras/MAPK pathway (Figure 6c). Therefore, gp130 cytokines regulate bone growth and bone remodeling by discrete activation of three pathways in three different cell lineages within the bone, and these distinct pathways may provide new avenues for improving bone growth and bone density.

\section{Acknowledgments}

The authors thank Ingrid Kriechbaum for expert histology, Jan Elliott for primary cell culture work, and Dianne Graill for excellent technical assistance. This work was supported by a Program Grant (003211) of the National Health and Medical Research Council, Australia, to M.T. Gillespie and T.J. Martin; N.A. Sims is funded by an NHMRC Career Development Award.

1. Ishihara, K., and Hirano, T. 2002. Molecular basis of the cell specificity of cytokine action. Biochim. Biophys. Acta. 1592:281-296.

2. Stahl, N., et al. 1994. Association and activation of Jak-Tyk kinases by CNTF-LIF-OSM-IL-6 $\beta$ receptor components. Science. 263:92-95.

3. Stahl, N., et al. 1995. Choice of STATs and other substrates specified by modular tyrosine-based motifs in cytokine receptors. Science. 267:1349-1353.

4. Nakajima, K., et al. 1996. A central role for Stat3 in IL-6-induced regulation of growth and differentiation in M1 leukemia cells. EMBO J. 15:3651-3658.
5. Fukada, T., et al. 1996. Two signals are necessary for cell proliferation induced by a cytokine receptor gp130: involvement of STAT3 in antiapoptosis. Immunity. 5:449-460.

6. Tebbutt, N.C., et al. 2002. Reciprocal regulation of gastrointestinal homeostasis by SHP2 and STAT-mediated trefoil gene activation in gp130 mutant mice. Nat. Med. 8:1089-1097.

7. Richards, C.D., Langdon, C., Deschamps, P., Pennica, D., and Shaughnessy, S.G. 2000. Stimulation of osteoclast differentiation in vitro by mouse oncostatin $\mathrm{M}$, leukaemia inhibitory factor, cardiotrophin-1 and interleukin 6: synergy with dexamethasone. Cytokine. 12:613-621.

8. Tamura, T., et al. 1993. Soluble interleukin-6 receptor triggers osteoclast formation by interleukin 6. Proc. Natl. Acad. Sci. U. S. A. 90:11924-11928.

9. Romas, E., et al. 1996. The role of gp130-mediated signals in osteoclast development: regulation of interleukin 11 production by osteoblasts and distribution of its receptor in bone marrow cultures. J. Exp. Med. 183:2581-2591.

10. Lowe, C., Cornish, J., Callon, K., Martin, T.J., and Reid, I.R. 1991. Regulation of osteoblast proliferation by leukemia inhibitory factor. J. Bone Miner. Res. 6:1277-1283.

11. Taguchi, Y., et al. 1998. Interleukin-6-type cytokines stimulate mesenchymal progenitor differentiation toward the osteoblastic lineage. Proc. Assoc. Am. Physicians. 110:559-574.

12. Heymann, D., and Rousselle, A.V. 2000. gp130 Cytokine family and bone cells. Cytokine. 12:1455-1468.

13. Takeuchi, Y., et al. 2002. Interleukin-11 as a stimulatory factor for bone formation prevents bone loss with advancing age in mice. J. Biol. Chem. 277:49011-49018.

14. Kitamura, H., et al. 1995. Bone marrow neutrophilia and suppressed bone turnover in human interleukin- 6 transgenic mice. A cellular relationship among hematopoietic cells, osteoblasts, and osteoclasts mediated by stromal cells in bone marrow. Am. J. Pathol. 147:1682-1692.

15. Ware, C.B., et al. 1995. Targeted disruption of the low-affinity leukemia inhibitory factor receptor gene causes placental, skeletal, neural and metabolic defects and results in perinatal death. Development. 121:1283-1299.

16. Sims, N.A., et al. 2002. Interleukin-11 receptor signaling is required for normal bone remodeling in male and female mice. J. Bone Miner. Res. 17:S143.

17. Kawasaki, K., et al. 1997. Osteoclasts are present in gp130-deficient mice. Endocrinology. 138:4959-4965.

18. Shin, H.I., et al. 2003. gp130-mediated signaling is necessary for normal osteoblastic function in vivo and in vitro. Endocrinology. doi:10.1210/en.2003-0839.

19. Hayashi, M., et al. 2002. Suppression of bone resorption by madindoline A, a novel nonpeptide antagonist to gp130. Proc. Natl. Acad. Sci. U. S. A. 99:14728-14733.

20. Ernst, M., et al. 2001. Defective gp130-mediated signal transducer and activator of transcription (STAT) signaling results in degenerative joint disease, gastrointestinal ulceration, and failure of uterine implantation. J. Exp. Med. 194:189-203.

21. Kopf, M., et al. 1994. Impaired immune and acute-phase responses in interleukin-6-deficient mice. Nature. 368:339-342.

22. Sims, N.A., et al. 2000. Bone homeostasis in growth hormone receptornull mice is restored by IGF-I but independent of Stat5. J. Clin. Invest. 106:1095-1103.

23. Glatt, M. 2001. The bisphosphonate zoledronate prevents vertebral bone loss in mature estrogen-deficient rats as assessed by micro-computed tomography. Eur. Cell. Mater. 1:18-26.

24. Quinn, J.M., Whitty, G.A., Byrne, R.J., Gillespie, M.T., and Hamilton, J.A. 2002. The generation of highly enriched osteoclast-lineage cell populations. Bone. 30:164-170.

25. Lam, J., Nelson, C.A., Ross, F.P., Teitelbaum, S.L., and Fremont, D.H. 2001. Crystal structure of the TRANCE/RANKL cytokine reveals determinants of receptor-ligand specificity. J. Clin. Invest. 108:971-979. doi:10.1172/JCI200113890.

26. Fujikawa, Y., Quinn, J.M., Sabokbar, A., McGee, J.O., and Athanasou, N.A. 1996. The human osteoclast precursor circulates in the monocyte fraction. Endocrinology. 137:4058-4060.

27. Montero, A., et al. 2000. Disruption of the fibroblast growth factor-2 gene results in decreased bone mass and bone formation. J. Clin. Invest. 105:1085-1093.

28. Sabatakos, G., et al. 2000. Overexpression of $\Delta$ FosB transcription factor(s) increases bone formation and inhibits adipogenesis. Nat. Med. 6:985-990.

29. Sims, N.A., et al. 2002. Deletion of estrogen receptors reveals a regulatory role for estrogen receptors-beta in bone remodeling in females but not in males. Bone. 30:18-25.

30. Sims, N.A., Morris, H.A., Moore, R.J., and Durbridge, T.C. 1994. Parathyroidectomy does not prevent bone loss in the oophorectomized rat. J. Bone Miner. Res. 9:1859-1863.

31. Kalu, D.N., Liu, C.C., Hardin, R.R., and Hollis, B.W. 1989. The aged rat model of ovarian hormone deficiency bone loss. Endocrinology. 124:7-16. 
32. Udagawa, N., et al. 1995. Interleukin (IL)-6 induction of osteoclast differentiation depends on IL-6 receptors expressed on osteoblastic cells but not on osteoclast progenitors. J. Exp. Med. 182:1461-1468.

33. Palmqvist, P., Persson, E., Conaway, H.H., and Lerner, U.H. 2002. IL-6, leukemia inhibitory factor, and oncostatin $\mathrm{M}$ stimulate bone resorption and regulate the expression of receptor activator of NF- $\mathrm{KB}$ ligand, osteoprotegerin, and receptor activator of $\mathrm{NF}-\kappa \mathrm{B}$ in mouse calvariae. J. Immunol. 169:3353-3362.

34. Nishimura, R., Moriyama, K., Yasukawa, K., Mundy, G.R., and Yoneda, T. 1998. Combination of interleukin- 6 and soluble interleukin- 6 receptors induces differentiation and activation of JAK-STAT and MAP kinase pathways in MG-63 human osteoblastic cells. J. Bone Miner. Res. 13:777-785.

35. Bellido, T., Borba, V.Z., Roberson, P., and Manolagas, S.C. 1997. Activation of the Janus kinase/STAT (signal transducer and activator of transcription) signal transduction pathway by interleukin-6-type cytokines promotes osteoblast differentiation. Endocrinology. 138:3666-3676.

36. Poli, V., et al. 1994. Interleukin-6 deficient mice are protected from bone loss caused by estrogen depletion. EMBO J. 13:1189-1196.

37. Manolagas, S.C., Kousteni, S., and Jilka, R.L. 2002. Sex steroids and bone. Recent Prog. Horm. Res. 57:385-409.

38. de Hooge, A.S., et al. 2003. Growth plate damage, a feature of juvenile idiopathic arthritis, can be induced by adenoviral gene transfer of oncostatin M: a comparative study in gene-deficient mice. Arthritis Rheum. 48:1750-1761.

39. Parfitt, A.M. 2002. Misconceptions (1): epiphyseal fusion causes cessation of growth. Bone. 30:337-339.

40. Martin, E.A., Ritman, E.L., and Turner, R.T. 2003. Time course of epiphyseal growth plate fusion in rat tibiae. Bone. 32:261-267.

41. Alonzi, T., et al. 1998. Interleukin 6 is required for the development of collagen-induced arthritis. J. Exp. Med. 187:461-468.

42. de Hooge, A.S., et al. 2002. Adenoviral transfer of murine oncostatin M elicits periosteal bone apposition in knee joints of mice, despite synovial inflammation and up-regulated expression of interleukin- 6 and receptor activator of nuclear factor- $\kappa B$ ligand. Am. J. Pathol. 160:1733-1743.

43. O’Brien, C.A., Gubrij, I., Lin, S.C., Saylors, R.L., and Manolagas, S.C. 1999. STAT3 activation in stromal/osteoblastic cells is required for induction of the receptor activator of NF- $\mathrm{KB}$ ligand and stimulation of osteoclastogenesis by gp130-utilizing cytokines or interleukin-1 but not 1,25-dihydroxyvitamin D3 or parathyroid hormone. J. Biol. Chem. 274:19301-19308.

44. Jenkins, B.J., et al. 2004. Imbalanced gp130-dependent signaling in macrophages alters M-CSF responsiveness via regulation of c-fms expression. Mol. Cell. Biol. 24. In press.

45. Gao, Y., et al. 1998. Expression of IL-6 receptor and GP130 in mouse bone marrow cells during osteoclast differentiation. Bone. 22:487-493.

46. Gouin, F., et al. 1999. Presence of leukaemia inhibitory factor (LIF) and LIF-receptor chain (gp190) in osteoclast-like cells cultured from human giant cell tumour of bone. Ultrastructural distribution. Cytokine. 11:282-289.

47. Girasole, G., Passeri, G., Jilka, R.L., and Manolagas, S.C. 1994. Interleukin-11: a new cytokine critical for osteoclast development. J. Clin. Invest. 93:1516-1524.

48. Hotokezaka, H., et al. 2002. U0126 and PD98059, specific inhibitors of MEK, accelerate differentiation of RAW2 64.7 cells into osteoclast-like cells. J. Biol. Chem. 277:47366-47372.

49. Wei, S., Wang, M.W., Teitelbaum, S.L., and Ross, F.P. 2002. Interleukin4 reversibly inhibits osteoclastogenesis via inhibition of NF- $\mathrm{KB}$ and mitogen-activated protein kinase signaling. J. Biol. Chem. 277:6622-6630.

50. Lee, S.E., et al. 2001. Tumor necrosis factor- $\alpha$ supports the survival of osteoclasts through the activation of Akt and ERK. J. Biol. Chem. 276:49343-49349.

51. Park, J.I., Strock, C.J., Ball, D.W., and Nelkin, B.D. 2003. The Ras/Raf/MEK/extracellular signal-regulated kinase pathway induces autocrine-paracrine growth inhibition via the leukemia inhibitory factor/JAK/STAT pathway. Mol. Cell. Biol. 23:543-554. 